\title{
An expert integrative approach for sediment load simulation in a tropical watershed.
}

\begin{abstract}
Prediction of highly non-linear behaviour of suspended sediment flow in rivers is of prime importance in environmental studies and watershed management. In this study, the predictive performance of artificial neural network (ANN) integrated with genetic algorithm (GA) was assessed. GA was used to optimize the parameters and architecture of the ANN. Five simulation scenarios (S1-S5) were developed using daily time series of suspended sediment discharge, water discharge, precipitation and reservoir level. The scenario S1 was composed of only water discharge input. The scenarios S2-S4 were composed of water discharge input and precipitation records at different stations. The inputs water discharge, precipitation and reservoir level formed the last scenario S5. Assessment metrics such as normalized mean square error, correlation coefficient, Nash-Sutcliffe efficiency and trend accuracy were used to evaluate the performance of ANN-GA on the simulation scenarios. Based on error analysis, differences between various scenarios in terms of error metrics were trivial, especially during the testing process. Meanwhile, S1 and S3 showed better accuracy in predicting the trend of sediment load time series, as compared to other scenarios. Based on error and sensitivity analyses, S1 with the Nash-Sutcliff efficiency and correlation coefficient of 0.56 and 0.81 , respectively, was chosen as the most appropriate scenario. All networks showed a weak robustness in estimating large magnitudes of sediment load, mostly attributable to scarcity of large observed values in the training data-set. This weakness was also originated from different non-linear relationships governing the process of sediment detachment and final sediment load by a high storm event, as compared to those by low or medium storm events. Furthermore, there was an obvious sediment load overestimation in the 2008 exemplars due to a high level of daily water discharge and the outlined generalization rules. Nevertheless, ANN-GA showed reliable performance for sediment load simulation in the studied watershed.
\end{abstract}

Keyword: Artificial neural network; Genetic algorithm; Sediment load; Hulu Langat watershed. 